\title{
Rehabilitation Therapy Intervention to Reduce Inflammation of Infected Surgical Incision
}

HONGXIA WANG AND XIAOYING BAO ${ }^{*}$

Operating Room, ${ }^{1}$ Outpatient Service, Affiliated Hospital of Gansu University of Chinese Medicine, Lanzhou 730020, China 
In order to explore the best treatment and rehabilitation outcome of post-infection inflammation by reducing wound infection and postoperative complications, 54 patients undergoing general surgery are divided into 2 groups to investigate rehabilitation intervention in the treatment of Infection of surgical incision. Results showed that there was no significant difference in operation time and neck drainage volume between the experimental group and the control group. C-reactive protein level of the experimental group was lower than that of the control group on days 1 and 3 after surgery. The $C$-reactive protein levels of the experimental group was lower than that of the control group at 12, 24, 48 and $48 \mathrm{~h}$ after operation. There was no significant difference in the pain score between the experimental group and the control group at $72 \mathrm{~h}$ after operation. The anxiety score of the experimental group was lower than that of the control group before and after operation and the satisfaction of the experimental group was higher than that of the control group. In the control group, in terms of hospitalization costs and hospitalization time, the experimental group was lower than the control group and the therapeutic effect of inflammatory infection was lower than that of the experimental group. Rehabilitation intervention needed to be carried out during the treatment of surgical incision infection and inflammation. It could reduce physical and mental discomfort of the patients and allow quick recovery as well as relieve the pain after operation, alleviate anxiety, and improve the patient satisfaction, shorten hospitalization time and reduce hospitalization expenses.

Key words: Rehabilitation therapy, CRP, inflammatory infection, statistical difference

Surgical site infection (SSI ${ }^{[1]}$ refers to incision-related infections that occur within $30 \mathrm{~d}$ after implant-free surgery or within $1 \mathrm{y}$ after implant-free surgery (such as pedicle screw, intervertebral fusion cage, artificial intervertebral disc) ${ }^{[2]}$. It often has a huge impact on the physical and mental health of patients. Postoperative wound infection not only endangers the health of patients, but also brings many social and economic problems, resulting in a significant increase in the average cost of treatment. It has been reported that the cost of treatment for patients with spinal infection is more than 4 times that of patients without infection ${ }^{[3,4]}$. Increased costs include secondary or multiple surgical costs, antibiotic treatment costs, ancillary examination costs and nutritional support costs, and early diagnosis of incision infection after surgery is quite difficult. The determination of inflammation-related blood indicators is the main method for early diagnosis of incision infection after operation ${ }^{[5]}$. At present, commonly used inflammation-related blood indicators include white blood cell count (WBC) and classification, erythrocyte sedimentation rate, C-reactive protein (CRP), procalcitonin, serum amyloid substance ${ }^{[6,7]}$.

In recent years, with advances in medical technology, a new bio-social-psycho-medical model has been formed. Of course, the purpose of developing this kind of model is not only to make the surgery successful, eliminate the disease and restore health, but also to minimize the trauma of patients, so that they can recover quickly. However, with the change of people's health concept and the enhancement of service consciousness, patients not only need a cure, but also high-quality services and rapid rehabilitation ${ }^{[8]}$. In this context, rehabilitation therapy intervention comes into main focus. At the beginning of this century, it was put forward that the meaning of rehabilitation therapy intervention refers to the cooperation of surgeons, anesthesiologists, nurses and other skilled personnel. In the process of surgery, scientific and effective methods are adopted to reduce patients' stress response level and prevent the operation from causing too much trauma to patients, so that the patients can recover quickly under the high-quality nursing service and the treatment outcome of diseases can be improved ${ }^{[9,10]}$.

According to the changes of inflammation index, the diagnosis of incision infection after operation is often speculative. In view of this phenomenon, changes of inflammatory indicators in patients after different operations are retrospectively analyzed, their changes are dynamically tracked and the data of patients in different groups are compared. The changes of inflammation-related blood indicators in the diagnosis of incision infection after surgery are compared, so as to judge the obvious degree of the effect of rehabilitation intervention on the inflammation of surgical incision infection.

Fifty four patients with inflammatory surgical incision infection and CRP markers in the Affiliated Hospital Gansu University of Chinese Medicine from June 2016 to June 2018 were selected comprising of 40 females

*Address for correspondence

E-mail: baoxiaoying1e32@163.com 
and 14 males, aged 32-70 y with an average age of $49.7 \pm 13.6$ y.

The inclusion criteria were, age range of 18 to $70 \mathrm{y}$. those who met the indications of operation and without any contraindications to the surgery. Patients with good cardiopulmonary function, without hypertension, diabetes or other complications and those with no local infection and other phenomena. The most important thing was that patients voluntarily participate and sign the informed consent. Exclusion criterion included, Age below 18 or over 70, pregnant or lactating women, patients with serious pathological changes in heart, brain, kidney and other important organs, or with recent onset of psychiatric disorders or language disorders and patients with previous neck surgery or radiation history.

The collected cases are numbered according to the length of hospitalization and then randomly divided into odd control group (24 cases) and even experimental group (30 cases). All patients underwent ultrasound examination, physical function examination, rapid frozen section examination during operation and pathological diagnosis after operation. Two groups of patients were subjected to the same operation, anesthesia and nursing team. The control group that developed surgical incision infection and inflammation were treated with traditional perioperative nursing measures, while the experimental group were provided rapid rehabilitation intervention measures. This study is a clinical randomized controlled trial approved by the ethics committee of the hospital. Through randomized grouping, the outcome of rehabilitation therapy intervention in patients with surgical incision infection and inflammation is addressed. The research protocol were shown in fig. 1.

All patients have blood pressure below 160/110 $\mathrm{mmHg}$, fasting blood sugar below $11.2 \mathrm{mM}$, and plasma albumin above $30 \mathrm{~g} / \mathrm{l}$. Anemia patients are corrected by plasma or red blood cells infusion before operation, and patients with smoking history quit smoking before operation. Antiplatelet drugs are discontinued $10 \mathrm{~d}$ before operation, and non-steroidal antiinflammatory drugs and cortisol were discontinued 2-3 d before operation. Fasting WBC count, neutrophil count (N), lymphocyte count (L) and CRP are measured 1 to 2 days before operation.

All the patients were given general anesthesia. Intravenous drip of a second generation cephalosporin (cefixime) was given $30 \mathrm{~min}$ before operation. For patients undergoing spinal canal decompression, methylprednisolone $120 \mathrm{mg}$ was given to prevent neuroedema during operation. One wound drainage tube is placed routinely in all patients. All patients with internal fixation were treated with $2 \mathrm{~g}$ QD of cefixime sodium, which is discontinued $72 \mathrm{~h}$ later. For patients with spinal canal decompression, the dosage of dexamethasone $5 \mathrm{mg}$ BID is reduced to $5 \mathrm{mg}$ QD for $3 \mathrm{~d}$ after operation and discontinued on the day 6 after operation. When the drainage volume is less than $50 \mathrm{ml}$ within $24 \mathrm{~h}$, the drainage tube should be removed.

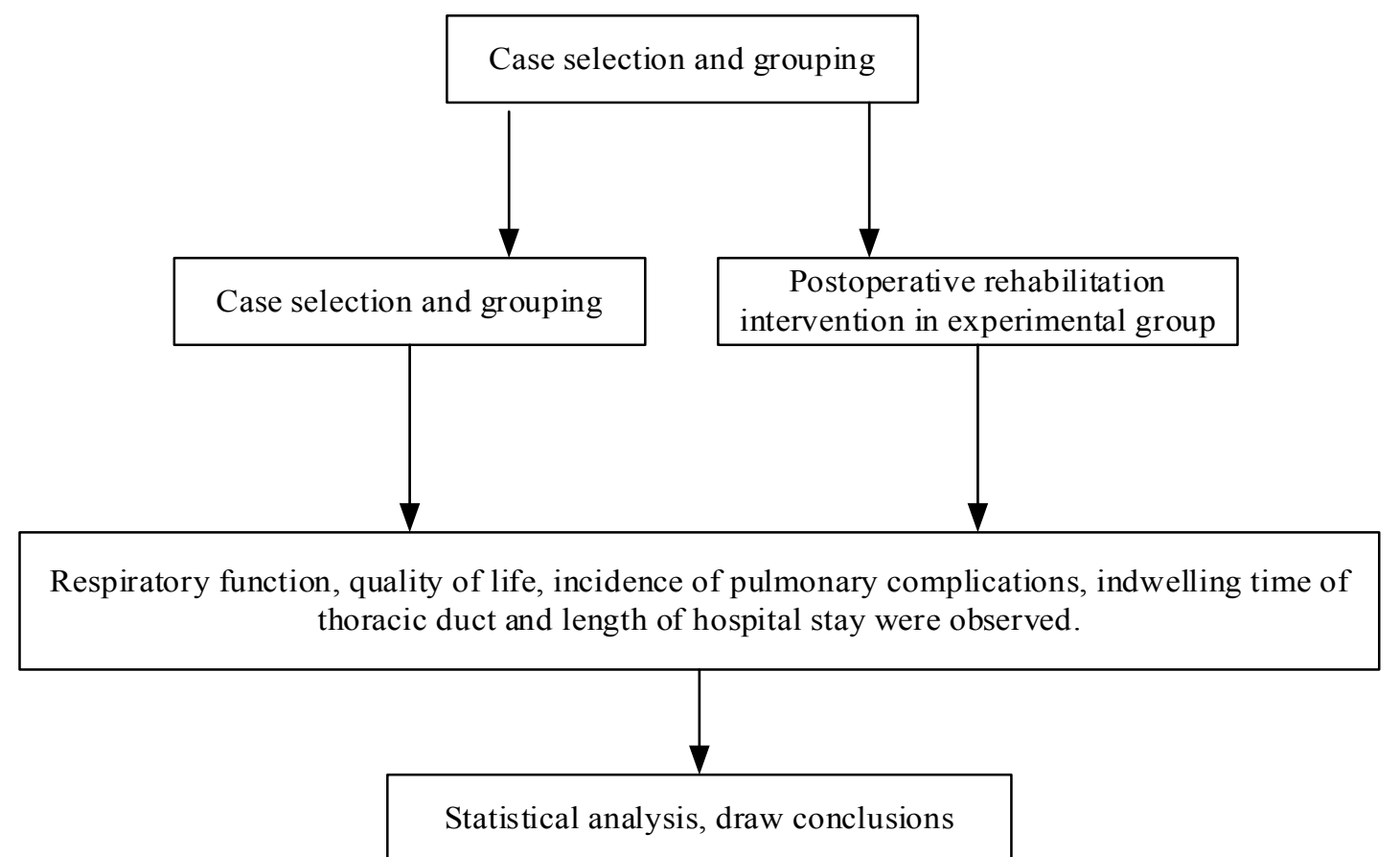

Fig. 1: Research protocol of rehabilitation intervention on inflammation of surgical incision infection 
Fasting WBC, N, L and CRP were measured on d 1, d 2-3, d 4-6 and d 7-9 after operation. Among them, WBC, $\mathrm{N}$ and $\mathrm{L}$ counts were measured by routine blood test, and CRP was measured by a biochemical test. Statistical analysis is made on the relationship between the above data and surgical incision infection. Rehabilitation interventions are formulated for the causes of surgical incision infection.

Rehabilitation intervention was initiated when infection occurred in the incision after operation and the patient is fully awake. Firstly, body position guidance was carried out from the first day after operation. Lateral decubitus position, in which the affected side allowed to lie on the upper side while the waist cushioned on a soft pillow. Both hips and knees flex. Relaxing the abdominal muscles could facilitate abdominal breathing. Pneumonectomy patients were made to avoid excessive lateral decubitus position, but positioned at 1/4 lateral decubitus position. Semi-prone position was that the upper half-prone position of the affected side is taken with a soft pillow padding under the chest, the lower leg is stretched, and the upper leg is flexed. Semi supine position was that in which the upper half supine position of the affected side is taken, and the pillow is cushioned on the back. Upper limb naturally falls back and lower limb flexion is placed beside the body. The lower limbs were in the same position as the lateral position. The above positions are carried out in turn. Each position lasted for $20 \mathrm{~min}$ to $1 \mathrm{~h}$ each time, twice a day.

Second, abdominal breathing, lip contraction breathing and effective cough and expectoration was applied. The patient was assisted to take a comfortable supine position or remain in a comfortable semi-supine position, with soft pillows under the knees to relax the abdominal muscles and the whole body of the patient. Researchers assisted patients in braking thoracic movement with one hand and abdominal pressure with the other hand. They instructed patients to alternately perform abdominal breathing, lip contraction breathing and effective cough and expectoration according to preoperative methods. On the day of operation, patients are assisted in one training session for $20 \mathrm{~min}$. On the first day after operation, patients were trained for $20 \mathrm{~min}$ in the morning and afternoon.

Third, lower limb exercises and out-of-bed training, in which after awakening, the patient was asked to move the lower limb joints in bed, flex the leg to stretch the leg and lift the leg. On the day after operation, the lower limbs are stretched forward alternately to prepare for walking. One group of 10 times is used to breathe in through the nose while stretching, while the lips are contracted and exhaled when lowering. After standing, patients were asked to walk step by step with the number of steps and time and walking distance increased daily. If a patient felt mild dyspnea he was asked to stop.

SPSS19.0 software (Windows) is used to analyze the data in both the experimental group and the control group. The data are displayed by mean \pm standard deviation, and $\mathrm{P}<0.05$ indicated significant difference. T-test was used to compare the mean of two independent samples. Variance analysis (F test) is used to compare the mean of multiple groups of data, and SNK-q test is used to compare the two groups.

CRP increases in the first few hours of inflammation and peaked at $48 \mathrm{~h}$. CRP decreases to normal level as the lesion subsided and tissue structure and function recovered. The response was not affected by radiotherapy, chemotherapy and corticosteroid therapy. Therefore, the detection of CRP has a wide range of clinical applications, including the diagnosis and differential diagnosis of acute infectious diseases, postoperative infection monitoring, observation of antibiotic efficacy, course detection and prognosis judgment. The degree of CRP elevation reflects the size or activity of inflammatory tissue. CRP has a good correlation with disease activity in acute inflammation and infection. This is not consistent with chronic inflammation. Although in some important cases, such as rheumatoid arthritis, segmental ileitis and rheumatic polymyalgia, this correlation is sufficient for therapeutic monitoring. CRP values of $10-50 \mathrm{mg} / \mathrm{l}$ indicate mild inflammation, such as local bacterial infections (cystitis, bronchitis, abscess), surgical and accidental trauma, myocardial infarction, deep venous thrombosis, inactive connective tissue disease, many malignant tumors and most viral sensations. The increase of CRP value to about $100 \mathrm{mg} / 1$ indicates more serious diseases, and the degree of inflammation needs intravenous injection when necessary. CRP value is more than $100 \mathrm{mg} / \mathrm{l}$, which indicates the process of serious diseases and often indicates the presence of bacterial infection.

The CRP of the two groups before, on the day after and $3 \mathrm{~d}$ after operation were measured and compared using sample $t$ test. The CRP of the experimental group and the control group before operation were within the normal range, while the CRP of the experimental group was $79.56 \pm 8.25$ on the day after surgery compared 
to that of the control group is $93.12 \pm 14.65$. Three days after surgery the CRP of the experimental group was $46.26 \pm 7.58$ and that of the control group was $54.28 \pm 10.04$. The CRP of the experimental group on the day after and $3 \mathrm{~d}$ after surgery was significantly lower than the corresponding levels of the control group ( $<<0.05$, fig. 2 ).

The pain scores of the two groups were evaluated within the time range of $12,24,48$ and $72 \mathrm{~h}$ after operation. The pain scores of the experimental and control groups were, $12 \mathrm{~h}$ after operation was $6.02 \pm 0.86$ and $7.97 \pm 0.46,24 \mathrm{~h}$ after surgery were $6.98 \pm 0.56$ and $9.12 \pm 1.36,48 \mathrm{~h}$ after surgery were $4.12 \pm 1.12$ and $5.72 \pm 1.68$, respectively. The pain scores of the experimental group at all these three time points were significantly lower than those of the control group. However $72 \mathrm{~h}$ after surgery the pain score in the experimental group was $1.78 \pm 0.67$, which was not significantly different from that of the control group's $2.47 \pm 0.93$ (fig. 3).

Increased incision contamination, increased incision length, unreasonable use of antibiotics, including invasive operations, are risk factors for nosocomial infections in patients after surgery, and the risk will continue to increase with the prolongation of the duration of invasive operations. Therefore, it can be seen that wound infection is the result of a combination of multiple causes, including the patient's own causes, medical personnel, articles and instruments, environmental health and other factors, so wound infection needs to be prevented in all aspects and links. Rehabilitation therapy intervention concept is a new concept based on medical needs. It combines the shortcomings of

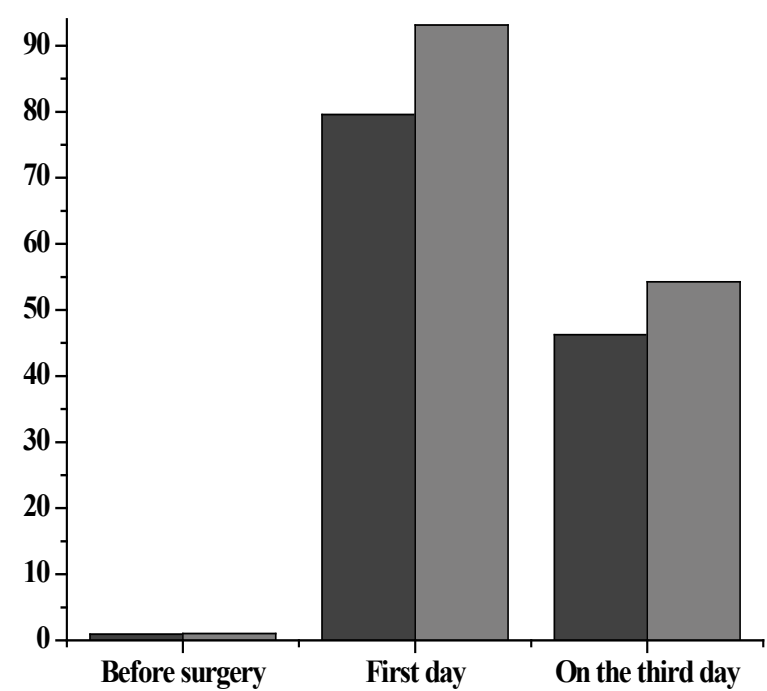

Fig. 2: Comparison of CRP between experimental group and control group

(匹) Experience group, ( $\square$ ) control group 85

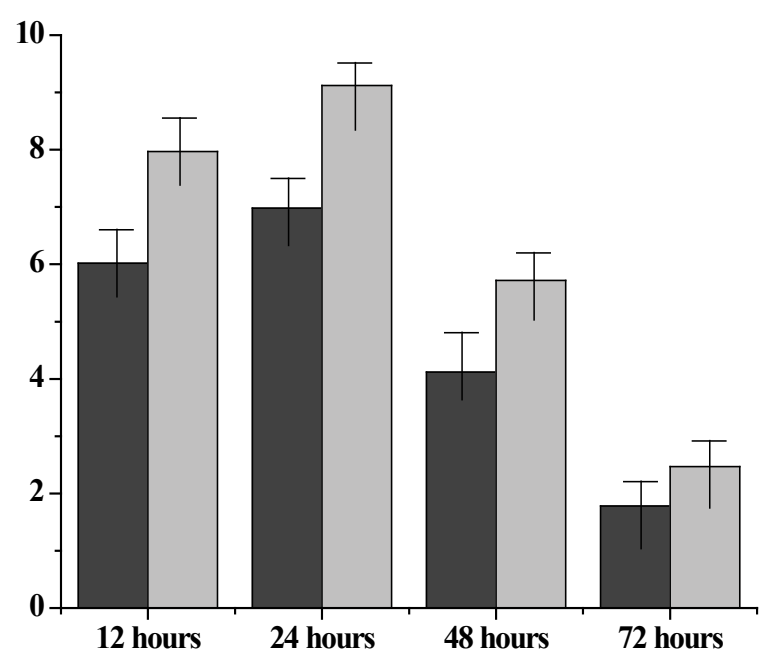

Fig. 3: Comparison of pain scores after operation between experimental group and control group

(a) Experience group, ( $\square$ ) control group

traditional medical care methods, and constantly carries out research around the real interests of patients, which greatly shortens the recovery time of patients after operation, thus effectively alleviating the pain and economic pressure of patients during hospitalization. Rehabilitation therapy intervention reduces the trauma and stress of patients during perioperative period, alleviates the pain of patients, increases the comfort of patients, and promotes early rehabilitation of patients. Compared with the traditional scheme, it also saves the hospitalization expenses, shortens the hospitalization time, and has good economic value.

In conclusion, CRP is a reliable indicator of the presence or absence of postoperative incision infection. Postoperative infection should be suspected when CRP increases twice after operation. Of course, after the use of rehabilitation therapy intervention, the number of CRP also means that rehabilitation intervention on surgical incision infection inflammation effect of the obvious degree. Compared with conventional treatment, rehabilitation intervention has obvious advantages.

\section{REFERENCES}

1. Edmiston CE, Spencer M. Patient Care Interventions to Help Reduce the Risk of Surgical Site Infections. AORN J 2014;100(6):590-602.

2. Johnson MP, Kim SJ, Langstraat CL. Using Bundled Interventions to Reduce Surgical Site Infection After Major Gynecologic Cancer Surgery. Obstet Gynecol 2016;127(6):1135-1144.

3. Schweizer ML, Chiang HY, Septimus E. Association of a bundled intervention with surgical site infections among patients undergoing cardiac, hip, or knee surgery. JAMA 2015;313(21):2162-2171.

4. Mcdonald LT, Clark AM, Landauer AK. Winning the War on Surgical Site Infection: Evidence ased Preoperative 
Interventions for Total Joint Arthroplasty. AORN J 2015;102(2):182-.

5. Mihaljevic AL, Schirren R, Müller TC. Postoperative negativepressure incision therapy following open colorectal surgery (Poniy): study protocol for a randomized controlled trial. Trials 2001;16(1):471.

6. Gillespie BM, Chaboyer W, Erichsen-Andersson A. Economic case for intraoperative interventions to prevent surgical-site infection. Br J Surg 2017; 104(2): e55.

7. Xie W, Su JH, Wang J. Changes of blood pressure, serum inflammatory factors and endothelin levels in patients with hypertension under rehabilitative aerobic exercise. J Biol Regul Homeost Agents 2019;33(2):531-536.

8. Sturm L, Flood M, Groves C. Bundled Interventions and Feedback on Performance Lead to Reductions of Colon and Hysterectomy Surgical Site Infections in a Large Health System. Am J Infect Control 2017;45(6):94.
9. Frenette C, Sperlea D, Tesolin J. Influence of a 5-year serial infection control and antibiotic stewardship intervention on cardiac surgical site infections. Am J Infect Control 2016;44(9):977-82.

10. Karl T, Reuss I, Schwab F. Reduction of inguinal surgical site infections after interventions on the lower extremities in vascular surgery. Gefässchirurgie 2017;22(38):1-9.

This is an open access article distributed under the terms of the Creative Commons Attribution-NonCommercial-ShareAlike 3.0 License, which allows others to remix, tweak, and build upon the work non-commercially, as long as the author is credited and the new creations are licensed under the identical terms

This article was originally published in Special issue on "Trends in therapeutic Management of Various Conditions" Indian J Pharm Sci 2020:82(3) 